\title{
Effects and Mechanism of Bufei Yishen Formula in a Rat Chronic Obstructive Pulmonary Disease Model
}

\author{
Jiansheng Li, ${ }^{1}$ Liping Yang, ${ }^{1}$ Qin Yao, ${ }^{2}$ Ya Li, $^{3}$ Yange Tian, ${ }^{3}$ Suyun Li, ${ }^{3}$ \\ Suli Jiang, ${ }^{3}$ Ying Wang, ${ }^{3}$ Xinmin Li, ${ }^{1}$ and Zheng Guo ${ }^{2,4}$ \\ ${ }^{1}$ Henan University of Traditional Chinese Medicine, Zhengzhou 450008, China \\ ${ }^{2}$ College of Bioinformatics Science and Technology, Harbin Medical University, Harbin 150086, China \\ ${ }^{3}$ Respiratory Disease Institute, The First Affiliated Hospital of Henan University of TCM, Zhengzhou 450000, China \\ ${ }^{4}$ Bioinformatics Centre, School of Life Science, University of Electronic Science and Technology of China, Chengdu 610054, China
}

Correspondence should be addressed to Jiansheng Li; jianshengli2013@126.com and Zheng Guo; guoz@ems.hrbmu.edu.cn

Received 17 January 2014; Revised 16 April 2014; Accepted 18 April 2014; Published 14 May 2014

Academic Editor: Syed Ibrahim Rizvi

Copyright (C) 2014 Jiansheng Li et al. This is an open access article distributed under the Creative Commons Attribution License, which permits unrestricted use, distribution, and reproduction in any medium, provided the original work is properly cited.

\begin{abstract}
Bufei Yishen Formula (BYF) has been used for centuries in Asia to effectively treat patients with chronic obstructive pulmonary disease (COPD). This study established a COPD animal model in rats, wherein three groups (control, COPD, and BYF) were used to evaluate the mechanism(s) and curative effect of BYF. Pulmonary function and histomorphology demonstrated that BYF had an evident effect on COPD. Gene microarray was then exploited to analyze the effects of BYF on COPD. ClueGO analysis of differentially expressed genes indicated that BYF improved COPD by regulating expression of interleukins, myosin filament assembly components, and mitochondrial electron transport-related molecules. Moreover, ELISA revealed that expression of several interleukins (IL1 $\beta, I L 6, I L 8$, and IL10) was reduced in peripheral blood and bronchoalveolar lavage fluid by BYF treatment. It was concluded that BYF has therapeutic effects on COPD in rats through its effects on interleukin expression and/or secretion. Furthermore, pharmacological or targeted expression of two differentially expressed genes, F2R and Sprikl, might be useful in novel COPD therapies. This study provides the basis for mechanisms of BYF on COPD and new therapeutic drug targets.
\end{abstract}

\section{Introduction}

Chronic obstructive pulmonary disease (COPD) is a slowly progressive, poorly reversible disease characterized by an abnormal inflammatory response in the lung [1]. The greatest risk factor in COPD development is cigarette smoking [1]. Other risk factors include air pollutants, dust, and inherent susceptibility [1]. The incidence of COPD in general populations is increasing, along with its great burden on public health $[2,3]$. Although some glucocorticoids and bronchodilators can alleviate acute onset of COPD, significant side effects exist [4]. For example, $\beta 2$ agonists can induce muscle tremor, tachycardia, sweats, and agitation [5]; theophylline can induce headache, nausea, vomiting, arrhythmias, and seizures [6]; and glucocorticoids can induce osteoporosis [7]. Therefore, the development of new longer lasting, targeted therapeutic strategies is a matter of great urgency.
At present, clinical trials and experimental studies have shown that certain Chinese medicines can effectively treat COPD, by improving pulmonary function, respiratory muscle fatigue, immunity, and lung blood flow [8-10]. Deng [11] found one Chinese herb formula that could improve COPD pathological presentation in a rat model, including inflammatory reactions and airway and pulmonary vasculature plasticity. Moreover, compared to western medicine, many Chinese herbs have few side effects [12]. However, the complexity and various actions of herbal components have limited their application and hindered study of their underlying mechanisms. With the development of highthroughput molecular techniques such as gene microarray, it has become possible to observe the effects of Chinese herbs on genomic expression and seek for their molecular targets.

As a traditional Chinese medicine formula, Bufei Yishen Formula (BYF) has been used for centuries in East Asia 


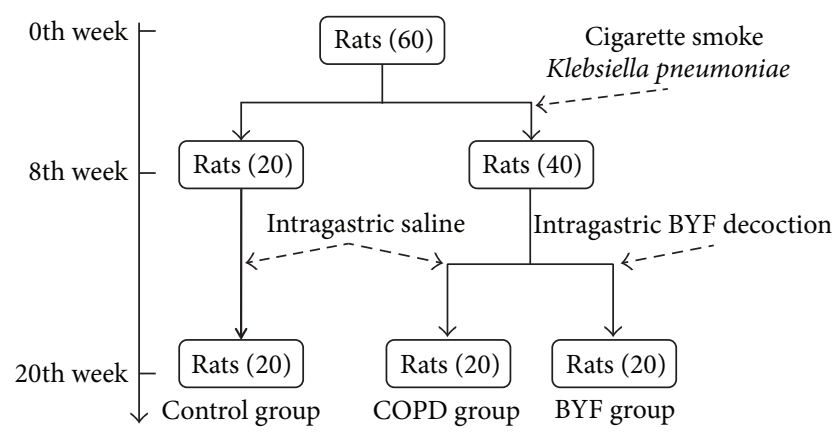

FIGURE 1: Establishment of COPD animal model.

to effectively treat patients with COPD. BYF principally employs the use of the herb Huang Qi (Radix Astragali) in its herbal formula. Multicentered clinical studies suggest that herbal formulae with Huang Qi were effective for stable COPD [13-18]. However, the quality of these studies has not been evaluated systematically, and no studies have yet explored the molecular mechanism of this particular herbal formula. Our previous studies have shown that BYF has more obvious beneficial effects on clinical symptoms of stable COPD than western medicine. Moreover, apparent positive long-term effects were observed for BYF treatment [19-21]. In this study, a modified COPD rat model was established as described previously by Chen et al. [22] for evaluating the effect(s) of BYF. Three experimental groups (COPD, BYF, and controls) were used to compare pulmonary function and pathology. To investigate the molecular mechanistic effect of BYF on COPD, differentially expressed genes were screened by microarray, and gene function enrichment analyses were conducted.

\section{Methods}

2.1. BYF Preparation and Animal Model Establishment. BYF was prepared at the First Affiliated Hospital of Henan University of Traditional Chinese Medicine (Zhengzhou, Henan, China). BYF components were comprised of $15 \mathrm{~g}$ Huang Qi (Radix Astragali), $15 \mathrm{~g}$ Ren Shen (Radix Ginseng), $15 \mathrm{~g}$ Shanzhuyu (Fructus Corni), and $9 \mathrm{~g}$ Wuweizi (Fructus Schisandrae). All herbs were decocted with water, steam sterilized, and brought to a final concentration of $0.6 \mathrm{~g} / \mathrm{mL}$. The BYF was supported by [9] and Chinese patent (number 2011101175781).

Experimental protocols were approved by the Experimental Animal Care and Ethics Committees of the First Affiliated Hospital, Henan University of Traditional Chinese Medicine. Two-month-old Sprague-Dawley rats were purchased from Henan Experimental Animal Center (Henan XK2005-0001) with a body weight of $200 \pm 20 \mathrm{~g}$. They were maintained on a 12-hour dark/light cycle with ambient temperature of $25 \pm 1^{\circ} \mathrm{C}$ and relative humidity $50 \pm 10 \%$, as well as sufficient food (sterile rat chow) and water (sterile). All rats were anesthetized and sacrificed under the experimental protocols mentioned above and all efforts were made to minimize suffering.
Sixty Sprague-Dawley rats were randomized and divided into three experimental groups (control, COPD, and BYF) (Figure 1) with equal numbers of males and females in each group. Forty rats underwent intranasal instillation with Klebsiella pneumoniae once every 5 days lasting for 8 weeks. Rats were placed in a $300 \mathrm{~L}$ smoke box for $30 \mathrm{~min}$ with $3 \mathrm{~h}$ intervals between smoke treatments, wherein eight cigarettes were burned twice daily in the first two weeks and 15 cigarettes were burned three times daily during weeks 38. After 8 weeks of treatment, the 40 COPD-induced rats were randomly divided into two groups: COPD and BYF. The COPD group was administered $2 \mathrm{~mL}$ intragastric saline vehicle $(0.9 \%)$ twice daily during weeks $9-20$, and the BYF group was administered $4.44 \mathrm{~g} / \mathrm{kg} / \mathrm{d}$ BYF twice daily during weeks 9-20. The remaining 20 rats were used as a control group with no treatment for the first 8 weeks followed by $2 \mathrm{~mL}$ intragastric saline vehicle $(0.9 \%)$ during weeks $9-$ 20. All rats were weighed weekly to determine dosing and underwent pathologic examination by lung tissue excision ( 6 samples/group) during the 21st week.

\subsection{Preparation and Determination of Pulmonary Function} and Pathology. Tidal volume (TV), peak expiratory flow (PEF), and 50\% tidal volume expiratory flow (EF50) were detected by unrestrained pulmonary function testing plethysmographs (Buxco Inc., Wilmington, NC, USA) conducted every fourth week from weeks 0 to 20. Paraffin-embedded sections of lung tissue were stained with hematoxylin and eosin and images were taken by light microscope (Olympus, Tokyo, Japan).

\subsection{Interleukin Detection in BALF and Peripheral} Blood. Following repeated saline bronchoalveolar lavage ( $3 \mathrm{~mL} /$ lavage), collected BALF underwent $10 \mathrm{~min}$ centrifugation $(2000 \mathrm{r} / \mathrm{min})$ and the supernatants were used to detect $I L 8$ and 10 by ELISA (BOSTER Inc., Wuhan, China). All processes were kept at $4^{\circ} \mathrm{C}$. Peripheral blood was collected from rat aorta abdominalis, and serum was used to detect ILI $\beta, I L 6, I L 8$, and IL10 by ELISA (BOSTER Inc.).

2.4. RNA Extraction. Six lung tissue samples were excised from each of the three experimental groups for microarray analysis. Total RNA was isolated from skeletal muscle by Trizol reagent (Invitrogen, Breda, Netherlands) and purified using a Qiagen RNeasy Micro kit (Qiagen, Venlo, Netherlands). RNA quality was verified by Agilent 2100 bioanalyzer (Agilent Technologies, Amsterdam, Netherlands).

2.5. Microarray Processing. Purified RNA samples ( $2 \mu \mathrm{g}$ ea $)$ were PCR amplified and labeled using an Agilent Quick Amp kit (Agilent Technologies, Santa Clara, CA, USA) and hybridized with Agilent Whole Rat Genome Oligo Microarray $(4 \times 44 \mathrm{~K})$ in Agilent's SureHyb Hybridization Chambers. After hybridization and washing, processed slides were scanned by an Agilent DNA microarray scanner (part number G2505B) using manufacturer recommended settings. 

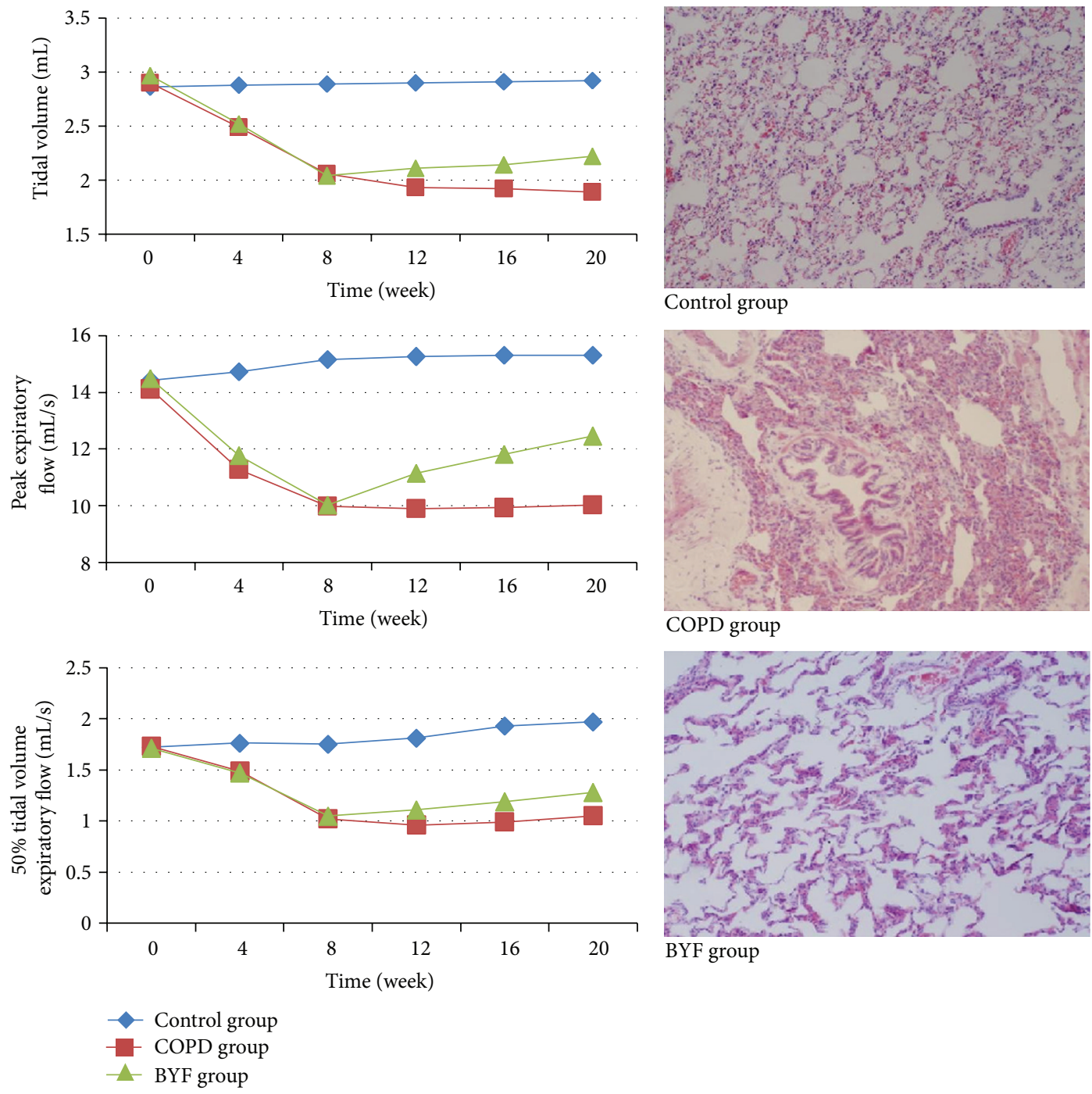

BYF group

(a)

(b)

FIGURE 2: Pulmonary function and pathology in each experimental rat group. (a) TV, PEF, and EF50. (b) Lung histomorphology observations by light microscope. Control group (magnification $\times 100$ ): pulmonary tissue was smooth and there was no inflammation and cell infiltration, no hyperemia, or no swelling. COPD group (magnification $\times 100$ ): pulmonary tissue showed upregulation of a severe inflammatory response with visible increases in lymphocytes, monocytes, and neutrophils. Bronchial and pulmonary wall thickness, degree of bronchial stenosis, and alveolar diameter were significantly higher. BYF group (magnification $\times 100$ ): inflammation around trachea lessened after BYF therapy. The number of inflammatory cells decreased dramatically.

TABLE 1: TV, PEF, and EF50 in three experimental rat groups $(\bar{x} \pm S)$.

\begin{tabular}{lccccccc}
\hline Index & Group & 0 weeks & 4 weeks & 8 weeks & 12 weeks & 16 weeks & 20 weeks \\
\hline \multirow{3}{*}{ TV $(\mathrm{mL})$} & Control & $2.86 \pm 0.46$ & $2.88 \pm 0.33$ & $2.89 \pm 0.34$ & $2.90 \pm 0.32$ & $2.91 \pm 0.36$ & $2.92 \pm 0.24$ \\
& COPD & $2.90 \pm 0.38$ & $2.49 \pm 0.24$ & $2.06 \pm 0.22$ & $1.93 \pm 0.22$ & $1.92 \pm 0.30$ & $1.89 \pm 0.36$ \\
& BYF & $2.97 \pm 0.46$ & $2.52 \pm 0.21$ & $2.04 \pm 0.33$ & $2.11 \pm 0.18$ & $2.14 \pm 0.25$ & $2.22 \pm 0.36$ \\
\hline \multirow{3}{*}{ PEF $(\mathrm{mL} / \mathrm{s})$} & Control & $14.43 \pm 2.69$ & $14.72 \pm 2.48$ & $15.16 \pm 1.99$ & $15.25 \pm 2.02$ & $15.31 \pm 1.75$ & $15.30 \pm 2.24$ \\
& COPD & $14.11 \pm 2.47$ & $11.28 \pm 2.00$ & $9.99 \pm 2.33$ & $9.90 \pm 1.96$ & $9.93 \pm 2.40$ & $10.02 \pm 2.18$ \\
& BYF & $14.49 \pm 2.53$ & $11.78 \pm 1.96$ & $10.03 \pm 2.48$ & $11.15 \pm 2.51$ & $11.81 \pm 2.61$ & $12.35 \pm 2.84$ \\
\hline \multirow{3}{*}{ EF50 (mL/s) } & Control & $1.72 \pm 0.16$ & $1.76 \pm 0.12$ & $1.75 \pm 0.15$ & $1.81 \pm 0.17$ & $1.93 \pm 0.28$ & $1.97 \pm 0.21$ \\
& COPD & $1.73 \pm 0.11$ & $1.49 \pm 0.13$ & $1.02 \pm 0.17$ & $0.96 \pm 0.20$ & $0.99 \pm 0.17$ & $1.05 \pm 0.17$ \\
& BYF & $1.71 \pm 0.12$ & $1.47 \pm 0.20$ & $1.05 \pm 0.20$ & $1.11 \pm 0.22$ & $1.17 \pm 0.28$ & $1.24 \pm 0.28$ \\
\hline
\end{tabular}




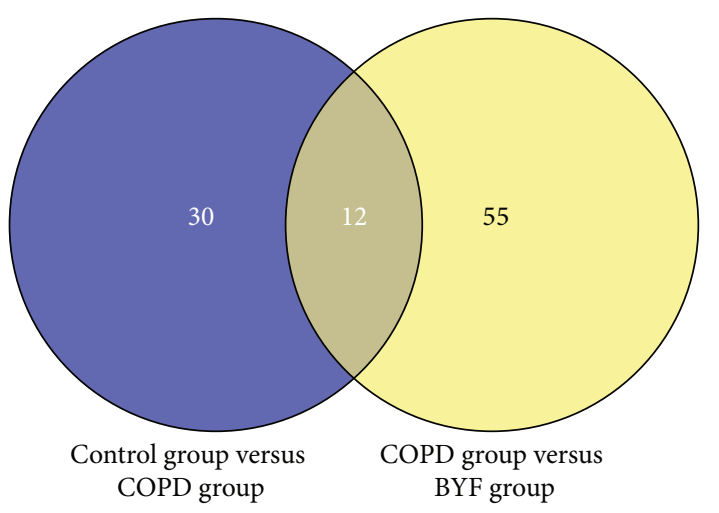

(a)

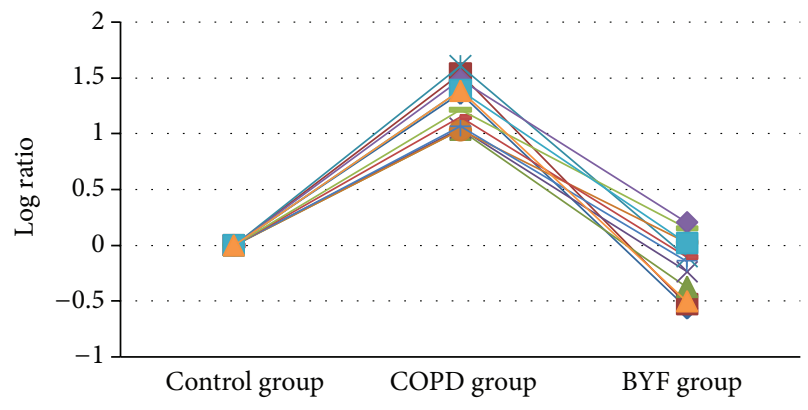

(b)

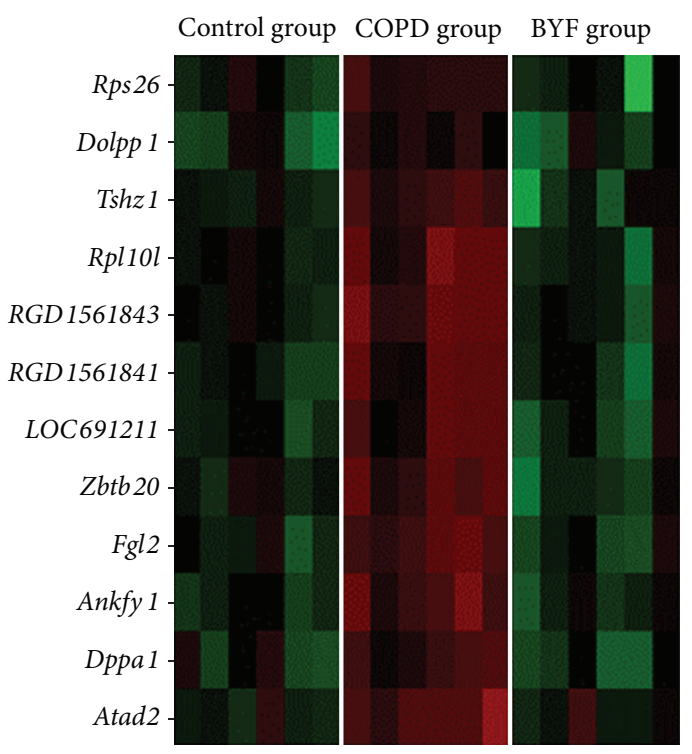

(c)

FIGURE 3: Venny plot of DEGs between each experimental group and expression of 12 overlapping genes. (a) Venny plot of two DEG lists (COPD versus controls and COPD versus BYF). (b) Expression of 12 overlapping genes from all three rat groups. (b) Gene expression heat map of 12 overlapping genes.

2.6. Data Preprocessing. Raw image data were converted to CEL and pivot files using Agilent Feature Extraction Software version 10.5.1.1. All downstream microarray analyses were performed using Agilent GeneSpring GX software version 11.0. Microarray datasets were normalized in GeneSpring GX using the Agilent FE one-color scenario (mainly median normalization); log 2-transformed data were normalized by quantile normalization and used for comparisons. Differentially expressed genes were identified through fold change $(\mid \log$ ratio $\mid>1)$ and Student's $t$-test screening $(P<0.05)$.

ClueGO software [24] was used for gene function enrichment analysis. We incorporated both gene ontology (GO) and the KEGG pathway in ClueGO analysis; GO term fusion and restriction with $P<0.05$ were chosen, which integrates GO categories and creates a functionally organized GO category network based on overlap between different GO categories and significance. Interleukin expression differences between groups were compared by Student's $t$-test $(P<0.01)$.

\section{Results}

3.1. Pulmonary Function and Pathology Improvements in COPD Rats with BYF Treatment. After 20 weeks, control group rats were active and restless, with smooth and burnished fur. Their body mass increased gradually and respiration remained stable. Before BYF treatment, rats in COPD and BYF groups exhibited "spiritual malaise," which is characterized by appetite suppression and wriggling with gathered fur in the first 8 weeks. The body mass of these rats slowly increased with short respiration accompanied by frequent cough. These symptoms in BYF group rats were significantly alleviated following BYF treatment from weeks 9 to 20 .

Pulmonary function for all three experimental groups was detected via TV, PEF, and EF50 every four weeks for 20 weeks. TV, PEF, and EF50 were found to be stable in the control group but were dramatically declined in the first eight 


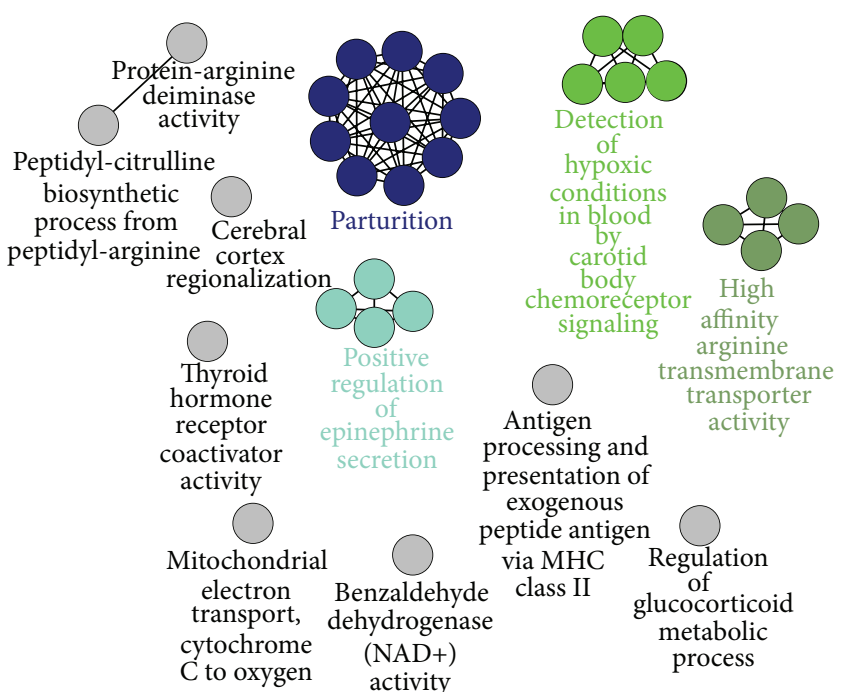

(a)

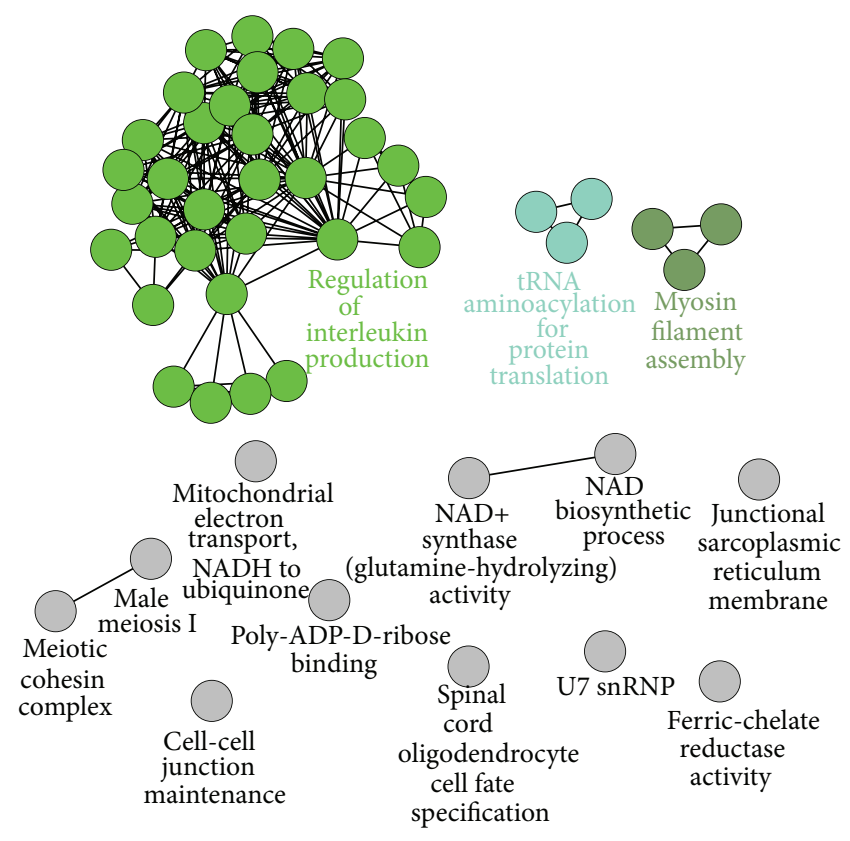

(b)

Figure 4: ClueGO analysis for DEGs. (a) ClueGO analysis for DEGs between COPD and control groups. (b) ClueGO analysis for DEGs between COPD and BYF groups.

weeks in the COPD and BYF groups $(P<0.05)$. Following BYF treatment, TV, PEF, and EF50 in the BYF group were significantly improved (all $P<0.05$; Table 1; Figure 2(a)) compared to rats of COPD group.

Lung histomorphology images from each of the three experimental groups are shown in Figure 2(b). Compared to controls, COPD rats showed upregulation of a severe inflammatory response with visible increases in lymphocytes, monocytes, and neutrophils. Bronchial and pulmonary wall thickness, degree of bronchial stenosis, and alveolar diameter were significantly higher in the COPD group, while the alveolar count per unit area was significantly lower compared to control rats. These COPD-related phenomena were dramatically relieved with BYF treatment. Furthermore, BYF treatment significantly alleviated the inflammatory response, as shown by a significant decrease in the number of inflammatory cells present in lung tissues.

3.2. Gene Microarray Data Analysis. To investigate the molecular mechanism of BYF on COPD pathogenesis, we randomly chose eighteen samples from the three experimental groups (six samples from each experimental group) for gene expression experiments. Using $\mid \log$ ratio $\mid>$ 1 and $P<0.05,42$, and 67, differentially expressed genes (DEGs) were detected between control and COPD groups as well as COPD and BYF groups (Figure 3(a); see Table S1 in Supplementary Material available online at http://dx.doi.org/10.1155/2014/381976), respectively. Twelve genes were overlapped between the two DEG lists (control versus COPD and COPD versus BYF). As shown in Figures $3(\mathrm{~b})$ and 3(c), all 12 overlapped genes were increased in COPD rats and then decreased in the BYF group. GO enrichment analysis by Bingo software [23] showed that these 12 overlapping genes were involved in ribosomal structure/function, gene expression, translation, and negative regulation of RNA splicing (Table 2). In particular, four overlapped genes (RPL10L, RPS26, RGD1561843, and RGD1561841) are located in the ribosome. This indicates that BYF treatment may reduce mRNA translation and translational editing in COPD.

3.3. ClueGO Analysis of Differentially Expressed Genes. To facilitate understanding of the biological implications of DEGs, functional enrichments were performed by ClueGO [24], which incorporates gene ontology and KEGG pathway annotation. ClueGO integrates GO categories and creates functionally organized GO category networks based on overlap between different GO categories and statistical significance. In line with previous studies of COPD and anoxic and oxidative stress [25], DEGs detected between our COPD and control groups were largely implicated in detection of hypoxic conditions in blood by carotid body chemoreceptor signaling, mitochondrial electron transport, cytochrome $\mathrm{C}$ to oxygen, and benzaldehyde dehydrogenase (NAD+) activity (Figure 4(a)). An especially interesting finding of this analysis was the significant enrichment for terms involved in parturition.

Significant GO and DEG pathway terms between COPD and BYF groups are presented in Figure 4(b). These DEGs were mainly enriched in terms involved in regulation of interleukin production, mitochondrial electron transport, $\mathrm{NADH}$ to ubiquinone, NAD+ synthase activity, NAD biosynthesis, poly-ADP-D-ribose binding, and myosin filament assembly. 

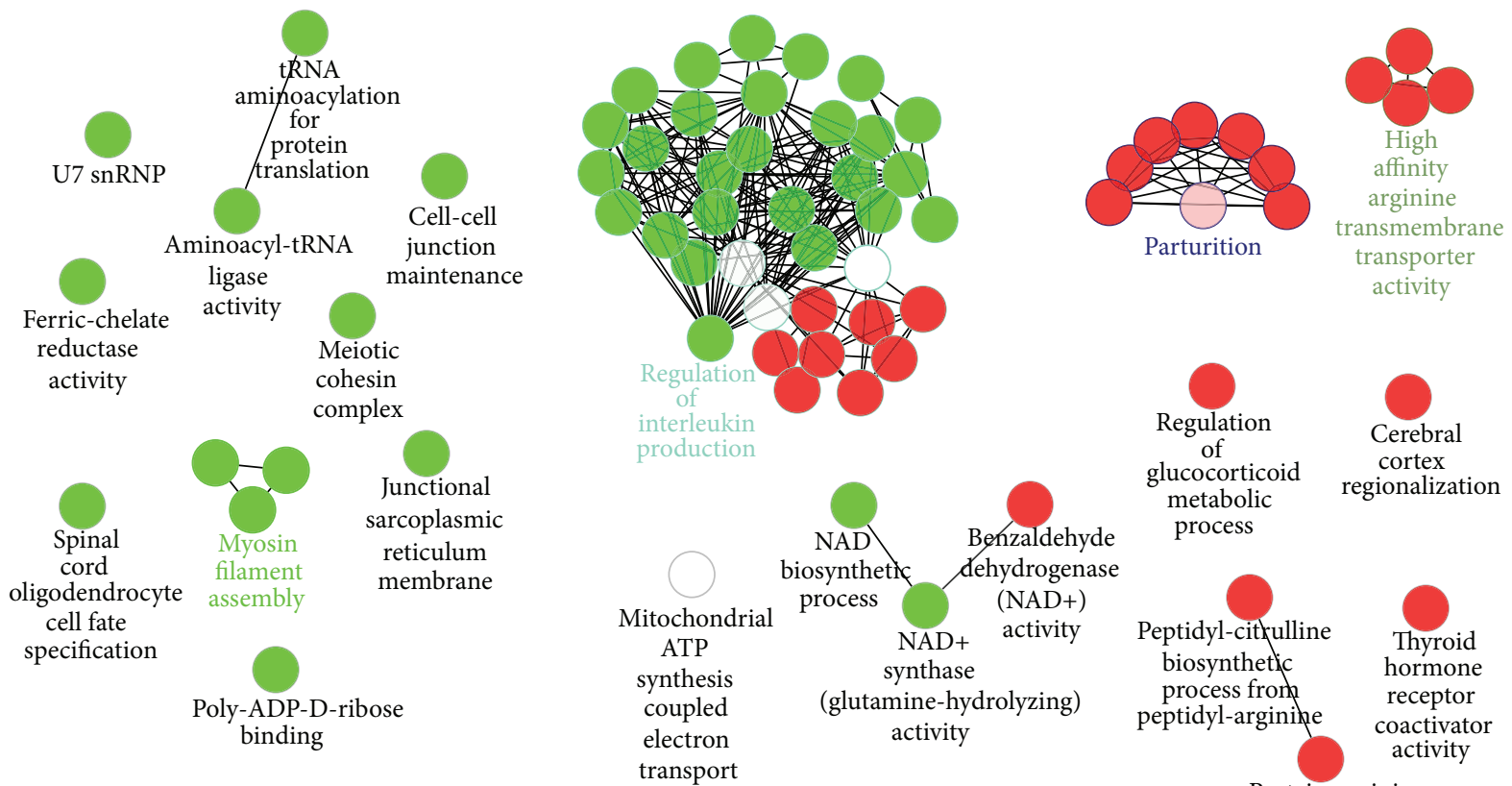

regionalization metabolic

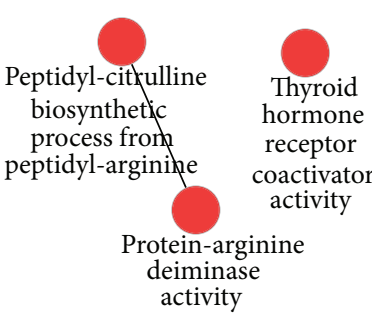

FIGURE 5: Comparative ClueGO analysis for two DEG lists (COPD versus controls and COPD versus BYF). Red node: functions only enriched by DEGs between control and COPD groups; green node: functions only enriched by DEGs between COPD and BYF groups; white node: functions enriched by both DEG lists.

TABLE 2: Bingo analysis of 12 overlapping genes [23].

\begin{tabular}{lccc}
\hline GO term & $P$ value & FDR & Gene \\
\hline Structural constituent of ribosome & $1.31 E-04$ & $1.16 E-02$ & $R P L 10 L / R P S 26 / R G D 1561843 / R G D 1561841$ \\
Ribosome & $1.76 E-04$ & $1.16 E-02$ & $R P L 10 L / R P S 26 / R G D 1561843 / R G D 1561841$ \\
Translation & $2.65 E-04$ & $1.17 E-02$ & $R P L 10 L / R P S 26 / R G D 1561843 / R G D 1561841$ \\
Ribonucleoprotein complex & $5.64 E-04$ & $1.86 E-02$ & $R P L 10 L / R P S 26 / R G D 1561843 / R G D 1561841$ \\
Structural molecule activity & $7.05 E-04$ & $1.86 E-02$ & $R P L 10 L / R P S 26 / R G D 1561843 / R G D 1561841$ \\
Soft palate development & $2.16 E-03$ & $3.55 E-02$ & $T S H Z 1$ \\
Negative regulation of RNA splicing & $2.16 E-03$ & $3.55 E-02$ & $R P S 26$ \\
Macromolecular biosynthesis & $2.42 E-03$ & $3.55 E-02$ & $R$ \\
Gene expression & $3.36 E-03$ & $4.44 E-02$ & $R$ RPL10L/RPS26/RGD1561843/RGD1561841 \\
\hline
\end{tabular}

Because COPD is known to be accompanied by pulmonary inflammation, oxidative stress, and muscle fiber dysfunction [25-27], BYF treatment may improve these COPD symptoms by regulating the above $\mathrm{GO}$ term functions.

Comparative ClueGO analysis between the two DEG lists (COPD versus control and COPD versus BYF) demonstrated that both DEG lists shared at least three relatively enriched GO terms in common including interleukin production, $\mathrm{NAD} / \mathrm{NADH}$, and mitochondrial electron transport-related function (Figure 5).

3.4. Interleukin Expression Level in Serum and BALF. Interleukin production and inflammatory functions were evaluated by ClueGO analysis [24], which demonstrated increases in the levels of several common serum (IL1 $\beta, I L 6, I L 8$, and
IL10) and BALF (IL8 and IL10) interleukins by ELISA. At the end of 20 weeks, serum levels of IL1 $\beta, I L 6, I L 8$, and IL10 in the COPD group were significantly higher than in the control group $(P<0.01)$, while those in the BYF group were significantly lower than the COPD group $(P<0.01)$. Similar to serum, IL8 and IL10 levels were also decreased with BYF treatment in BALF $(P<0.01$; Table 3; Figure 6(b)). These findings indicate that BYF can alleviate COPD inflammation by reducing interleukin levels.

\section{Discussion}

Although technological advances have been made, there are still considerable complications associated with human tissue sampling. Animal models that imitate COPD pathogenesis 
$I L 1 \beta(\mathrm{ng} / \mathrm{L})$

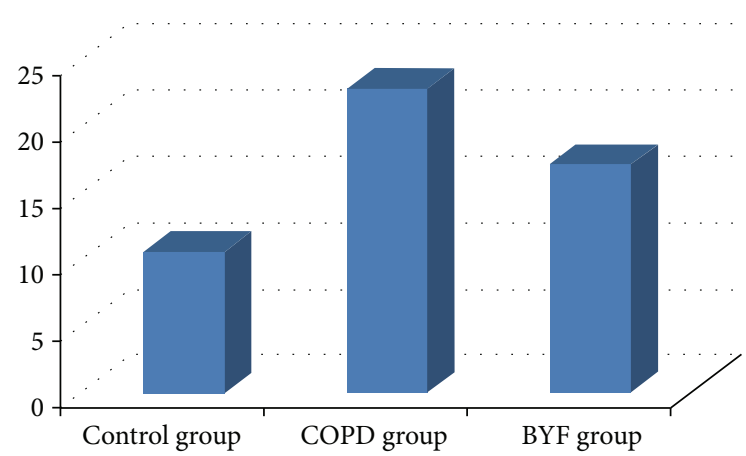

$I L 8(\mathrm{ng} / \mathrm{L})$

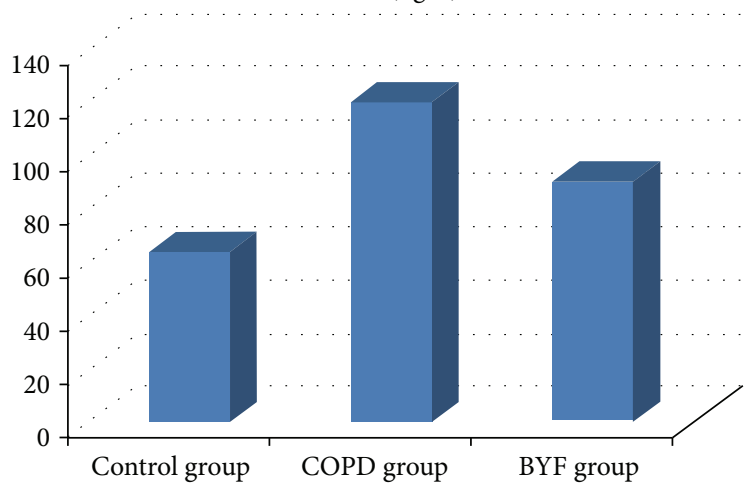

(a)

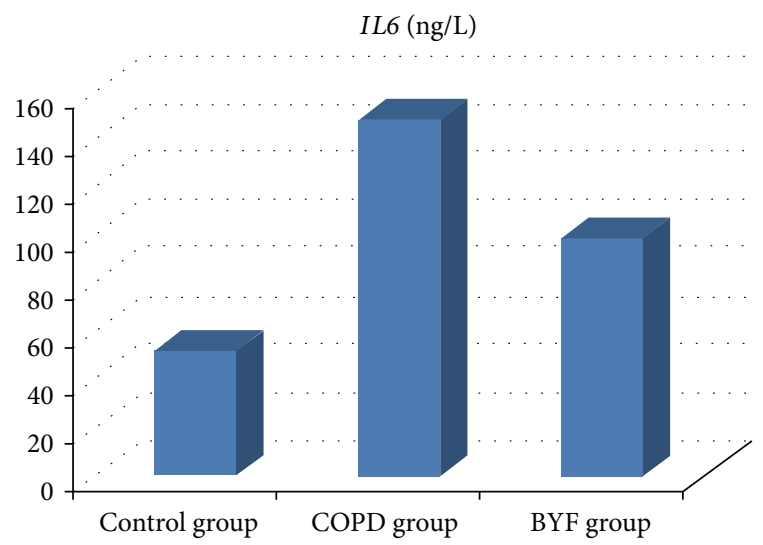

IL10 (ng/L)

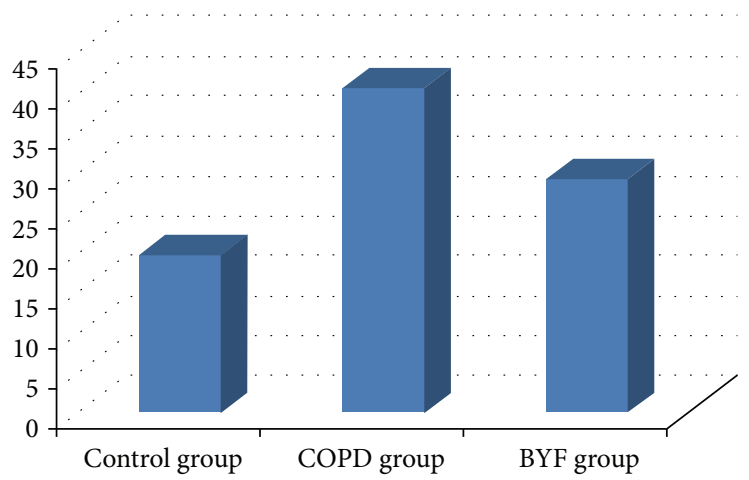

)
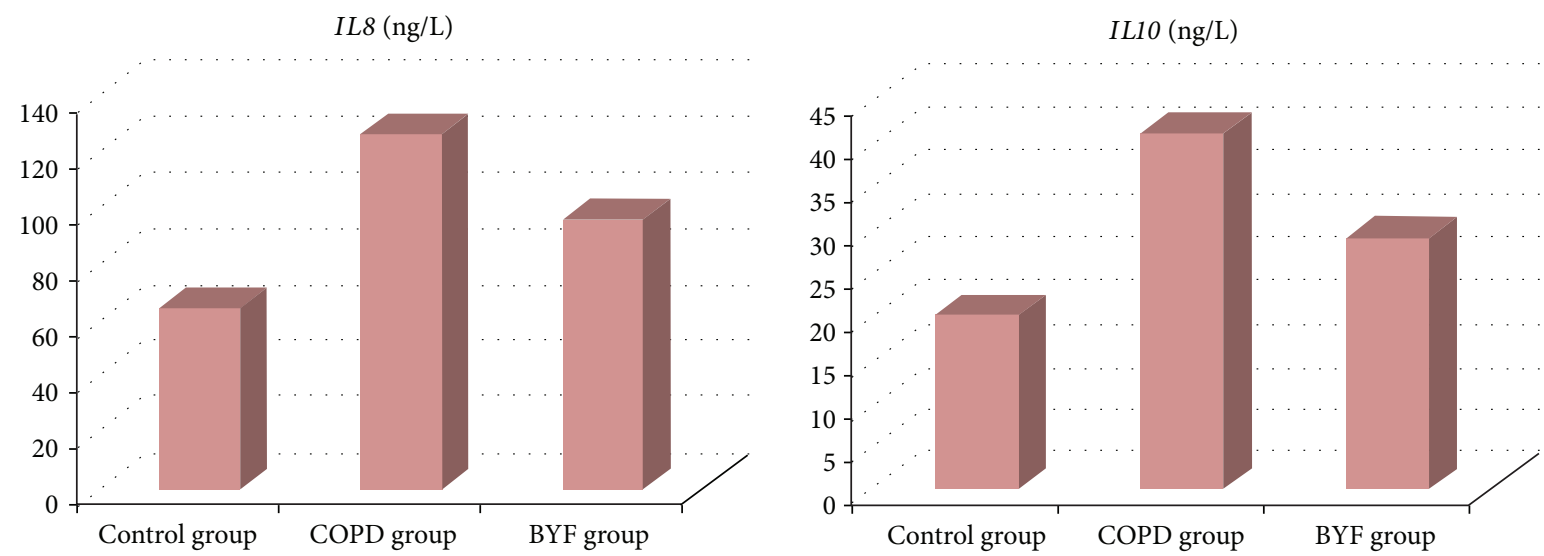

(b)

FIGURE 6: Interleukin expression levels in BALF and serum. (a) Expression level of IL1 $\beta, I L 6, I L 8$, and IL10 in serum. (b) Expression level of IL8 and IL10 in BALF.

avoid the risks of human experimentation [28-30] and provide a basis for evaluating the effectiveness of new therapeutic strategies. At present, COPD animal models that are consistent with human COPD are available $[9,10]$. In this study, rat COPD was induced by cigarette smoking in combination with repeated instillation of Klebsiella pneumonia. COPD lung tissue pathology by light microscope demonstrated that lesions in the rat model closely resemble those occurring in human COPD.
The complexity and varying action of components of Chinese herbs have limited their extensive utilization and hindered study of their underlying molecular mechanism(s). With development of high-throughput molecular techniques such as gene microarray, it is possible to examine the effects of Chinese herbs on genomic expression. To evaluate the effect of BYF on COPD in the present study, we established three groups of rats (COPD, BYF, and controls) and compared their pulmonary function and pathology. Results showed 


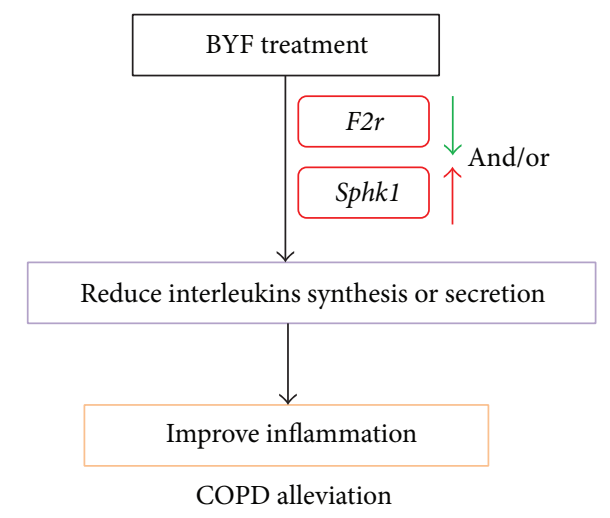

FIGURE 7: Mechanism of BYF treatment for COPD.

TABLE 3: Interleukin expression levels in serum and BALF in the 20th week.

\begin{tabular}{lccc}
\hline Interleukin & Group & Serum $(\mathrm{ng} / \mathrm{L})$ & BALF $(\mathrm{ng} / \mathrm{L})$ \\
\hline \multirow{4}{*}{ IL1 $\beta$} & Control & $10.62 \pm 1.68$ & \\
& COPD & $22.81 \pm 1.34$ & \\
& BYF & $17.13 \pm 0.71$ & \\
IL6 & Control & $52.48 \pm 3.42$ & \\
& COPD & $148.71 \pm 4.72$ & \\
\hline \multirow{3}{*}{ IL8 } & BYF & $99.32 \pm 4.45$ & \\
& Control & $64.06 \pm 8.17$ & $64.60 \pm 8.77$ \\
& COPD & $120.33 \pm 7.15$ & $126.60 \pm 6.89$ \\
IL10 & BYF & $90.33 \pm 4.37$ & $96.52 \pm 6.90$ \\
\hline \multirow{4}{*}{} & Control & $19.72 \pm 2.93$ & $20.18 \pm 2.43$ \\
& COPD & $40.50 \pm 2.13$ & $41.16 \pm 2.58$ \\
& BYF & $29.18 \pm 1.55$ & $29.12 \pm 1.54$ \\
\hline
\end{tabular}

that BYF treatment can significantly improve pulmonary function and lung tissue pathology of COPD rats. Gene expression profiles were used to explore the multitarget characteristics of BYF treatment. Gene function enrichment analysis indicated that the BYF can improve COPD through mitochondrial electron transport-related molecules (NAD/NADH/ADP), regulation of interleukin expression, and myosin filament assembly components, relating to muscle dysfunction. Many studies suggested that COPD was related to respiratory, diaphragmatic, and skeletal muscle dysfunction [27, 31-33]. Mitochondrial electron transportrelated molecules (NAD/NADH/ADP) are also in relation with redox reactions and oxidative stress, which are known to be the key factors in COPD $[33,34]$. In addition, regulation of interleukin expression, involved in inflammation, is also a symptom of COPD [35-37].

Chronic airway inflammation is a key aspect in the pathogenesis of COPD, associated with almost all structural and functional damage of airway and lung tissue [38]. Previous studies showed that members of inflammatory cells such as neutrophils, lymphocytes, and alveolar macrophages were aggregated in blood, sputum, BALF, and bronchial mucosa in stable COPD patients [39]. Inflammatory cells also release various cytokines, including IL1 $\beta, I L 6, I L 8$, and IL10, which play an important role in inflammatory response. In this study, we detected expression of IL1 $\beta, I L 6, I L 8$, and IL10 in peripheral blood and BALF. Results show that all four interleukins were reduced by BYF treatment in COPD rats, which was consistent with the gene function enrichment analysis.

In gene expression profiles, no difference was found for interleukin expression among treatment groups. However, expression changes of several interleukins were detected in BALF and serum, suggesting that BYF treatments cannot downregulate interleukin transcription but reduce translation and/or protein secretion. Gene function enrichment analysis of 12 overlapping DEGs (between control versus COPD and COPD versus BYF groups) indicates that BYF treatment can reduce translation and RNA splicing in COPD. As confirmation, we focused on two DEGs (F2R and Sphk1) that are related to the regulation of interleukin production. Interestingly, studies by Gigante et al. [40, 41] showed that F2R haplotypes influence serum IL6 levels in humans and regulate IL6 synthesis and production in endothelial cells. In other words, F2R haplotypes may influence IL6 synthesis and secretion. Besides, the human protein-protein interactions predictions (PIPs) database predicts that $F 2 R$ may physically interact with IL8 [42], while Li et al. [43] found that Sphk1 expression and activity could reduce IL1 $\beta$ and IL6 concentrations in the serum. Therefore, we speculate that $F 2 R$ and Sphkl can regulate the synthesis and/or secretion of certain interleukins in our COPD rat models, while BYF treatment does not influence transcription but reduces interleukin translation or secretion by regulating expression of F2R and Sphk1, thereby improving COPD-related inflammation (Figure 7).

\section{Conflict of Interests}

The authors declare that there is no conflict of interests regarding the publication of this paper.

\section{Authors' Contribution}

Jiansheng Li and Liping Yang contributed equally to the work.

\section{Acknowledgments}

The authors thank Zhang Yang for helping them to complete the bioinformatic analysis. This research was supported by the National Natural Sciences Foundation of China (nos. 81130062, 81173236, and 30973743).

\section{References}

[1] K. F. Rabe, S. Hurd, A. Anzueto et al., "Global strategy for the diagnosis, management, and prevention of chronic obstructive pulmonary disease: GOLD executive summary," The American Journal of Respiratory and Critical Care Medicine, vol. 176, no. 6, pp. 532-555, 2007.

[2] R. A. Pauwels, A. S. Buist, P. M. A. Calverley, C. R. Jenkins, and S. S. Hurd, "Global strategy for the diagnosis, management, and prevention of chronic obstructive pulmonary disease: 
National Heart, Lung, and Blood Institute and World Health Organization Global Initiative for Chronic Obstructive Lung Disease (GOLD): executive summary," Respiratory Care, vol. 46, no. 8, pp. 798-825, 2001.

[3] J. B. Soriano and R. Rodríguez-Roisin, "Chronic obstructive pulmonary disease overview: epidemiology, risk factors, and clinical presentation," Proceedings of the American Thoracic Society, vol. 8, no. 4, pp. 363-367, 2011.

[4] L. Wang, "Drug treatment and development of COPD," Chinese Journal of Urban and Rural Industrial Hygiene, vol. 6, no. 3, pp. 22-23, 2006.

[5] P. B. Wu and J. G. Hu, "Application of $\beta 2$ receptor agonists in the treatment of respiratory diseases," Journal of Clinical Pulmonary Medicine, vol. 15, no. 6, pp. 993-994, 2010.

[6] K. Ito, S. Lim, G. Caramori et al., "A molecular mechanism of action of theophylline: induction of histone deacetylase activity to decrease inflammatory gene expression," Proceedings of the National Academy of Sciences of the United States of America, vol. 99, no. 13, pp. 8921-8926, 2002.

[7] R. N. J. De Nijs, "Glucocorticoid-induced osteoporosis: a review on pathophysiology and treatment options," Minerva Medica, vol. 99, no. 1, pp. 23-43, 2008.

[8] M. Z. Guo, Z. X. Yang, and Y. Q. Xiao, "Development of the study on the effects of Chinese herbs in treating COPD," Chinese Medicine and Pharmacology, vol. 37, no. 5, p. 105, 2009.

[9] Q. Chen and S. M. Liu, "Study on the changes of blood rheology and the interfere effect of herbs with invigorating qi and promoting blood circulation effect in patients with chronic obstructive pulmonary disease," Hunan Journal of Traditional Chinese Medicine, vol. 21, no. 3, pp. 3-7, 2005.

[10] C. Y. Tang, L. Lin, and Y. J. Xu, "Effects of strengthening earth to support metal on nutrition and lung function in stable COPD patients," Journal of Nanjing University of Traditional Chinese Medicine, vol. 21, no. 3, article 16, 2005.

[11] L. Deng, Role of three regulating lung and kidney methods of inflammatory factor in lung tissue of rat with COPD and its remote effect [M.S. thesis], Henan University of Traditional Chinese Medicine, Zhengzhou, China, 2011.

[12] G. Xiong, S. Chen, W. Xie, X. D. Ye, and J. Lin, "Effects of Shenge Powder on lung function, the level of serum IL-8, TNF- $\alpha$ in stable COPD," Chinese Journal of Geriatric Care, vol. 6, no. 4, pp. 37-39, 2008.

[13] S. Y. Li, J. S. Li, L. J. Ma, Q. W. Zhou, and C. H. Li, "Effects of Lung-tonifying and Kidney-strengthening Capsule on adhesion molecules in stable COPD patients," China Journal of Traditional Chinese Medicine and Pharmacy, vol. 18, no. 1, article 500, 2003.

[14] H. L. Zhang, Efficacy of patients with stable chronic obstructive pulmonary disease by differentiation treatment and the influence of systemic inflammatory response with traditional Chinese medicine [M.S. thesis], Henan University of Traditional Chinese Medicine, Zhengzhou, China, 2010.

[15] L. S. Xu, "The influence of combined treatment of traditional Chinese medicine and western medicine on pulmonary function with the patients of moderate and severe COPD," China and Foreign Medical Journal, vol. 5, no. 2, pp. 55-56, 2007.

[16] M. L. Hong, L. Y. Gao, and S. Z. Dai, “The efects of Yufeining on respiratory muscle strength and central respiratory drive in patients with COPD," Chinese Journal of Information on Traditional Chinese Medicine, vol. 11, no. 11, pp. 961-963, 2004.

[17] G. L. Jia, F. J. Tong, D. Z. Yu et al., "The clinical study of Yiqihuoxue combined ipratropium bromide inhalation in the treatment of chronic obstructive pulmonary disease," Zhejiang Journal of Traditional Chinese Medicine, vol. 42, no. 9, pp. 510511, 2007.

[18] S. Wang, H.-Y. Ji, N.-Z. Zhang et al., "Effect of yifei jianpi recipe on inflammatory cells, levels of interleukin-8 and tumor necrosis factor-alpha in sputum from patients with chronic obstructive pulmonary disease," Chinese Journal of Integrated Traditional and Western Medicine, vol. 25, no. 2, pp. 111-113, 2005.

[19] S. Y. Li, J. S. Li, M. H. Wang et al., "Effects of comprehensive therapy based on traditional Chinese medicine patterns in stable chronic obstructive pulmonary disease: a four-center, openlabel, randomized, controlled study," BMC Complementary and Alternative Medicine, vol. 12, no. 1, article 197, 2012.

[20] J.-S. Li, Y. Li, S.-Y. Li et al., "long-term effect of Tiaobu Feishen therapies on systemic and local inflammation response in rats with stable chronic obstructive pulmonary disease," Zhong Xi Yi Jie He Xue Bao, vol. 10, no. 9, pp. 1039-1048, 2012.

[21] S. Y. Li, Y. Li, J. S. Li et al., "Efficacy and long-term effect of three therapies of invigorating lung and kidney for rat with stable COPD," China Journal of Traditional Chinese Medicine and Pharmacy, vol. 27, no. 12, pp. 3116-3120, 2012.

[22] P. R. Chen, L. Cui, and J. F. Hou, "Advances in research on development of animal models of chronic obstructive pulmonary disease," Acta Laboratorium Animalis Scientia Sinica, vol. 15, no. 5, pp. 238-242, 2007.

[23] S. Maere, K. Heymans, and M. Kuiper, "BiNGO: a Cytoscape plugin to assess overrepresentation of Gene Ontology categories in Biological Networks," Bioinformatics, vol. 21, no. 16, pp. 34483449,2005

[24] G. Bindea, B. Mlecnik, H. Hackl et al., "ClueGO: a Cytoscape plug-in to decipher functionally grouped gene ontology and pathway annotation networks," Bioinformatics, vol. 25, no. 8, pp. 1091-1093, 2009.

[25] D. T. Stuss, I. Peterkin, D. A. Guzman, C. Guzman, and A. K. Troyer, "Chronic obstructive pulmonary disease: effects of hypoxia on neurological and neuropsychological measures," Journal of Clinical and Experimental Neuropsychology, vol. 19, no. 4, pp. 515-524, 1997.

[26] W. Q. Gan, S. F. P. Man, A. Senthilselvan, and D. D. Sin, "Association between chronic obstructive pulmonary disease and systemic inflammation: a systematic review and a metaanalysis," Thorax, vol. 59, no. 7, pp. 574-580, 2004.

[27] C. A. C. Ottenheijm, L. M. A. Heunks, and P. N. R. Dekhuijzen, "Diaphragm muscle fiber dysfunction in chronic obstructive pulmonary disease: toward a pathophysiological concept," The American Journal of Respiratory and Critical Care Medicine, vol. 175, no. 12, pp. 1233-1240, 2007.

[28] J. L. Wright, M. Cosio, and A. Churg, "Animal models of chronic obstructive pulmonary disease," The American Journal of Physiology_Lung Cellular and Molecular Physiology, vol. 295, no. 1, pp. L1-L15, 2008.

[29] J.-H. Lee, D.-S. Lee, E.-K. Kim et al., "Simvastatin inhibits cigarette smoking-induced emphysema and pulmonary hypertension in rat lungs," The American Journal of Respiratory and Critical Care Medicine, vol. 172, no. 8, pp. 987-993, 2005.

[30] H. A. Zheng and S. H. He, "Progress in methods to produce COPD animal model," Acta Laboratorium Animalis Scientia Sinica, vol. 11, no. 4, pp. 249-252, 2003.

[31] H. C. Kim, M. Mofarrahi, and S. N. A. Hussain, "Skeletal muscle dysfunction in patients with chronic obstructive pulmonary 
disease," International Journal of COPD, vol. 3, no. 4, pp. 637658, 2008.

[32] M. J. Mador and E. Bozkanat, "Skeletal muscle dysfunction in chronic obstructive pulmonary disease," Respiratory Research, vol. 2, no. 4, pp. 216-224, 2001.

[33] L. Zuo, A. H. Hallman, M. K. Yousif, and M. T. Chien, "Oxidative stress, respiratory muscle dysfunction, and potential therapeutics in chronic obstructive pulmonary disease," Frontiers in Biology, vol. 7, no. 6, pp. 506-513, 2012.

[34] A. G. D. M. Cavalcante and P. F. C. de Bruin, "The role of oxidative stress in COPD: current concepts and perspectives," Jornal Brasileiro de Pneumologia, vol. 35, no. 12, pp. 1227-1237, 2009.

[35] E. F. Wouters, "Local and systemic inflammation in chronic obstructive pulmonary disease," Proceedings of the American Thoracic Society, vol. 2, no. 1, pp. 26-33, 2005.

[36] S. Sethi, D. A. Mahler, P. Marcus, C. A. Owen, B. Yawn, and S. Rennard, "Inflammation in COPD: implications for management," The The American Journal of Medicine, vol. 125, no. 12, pp. 1162-1170, 2012.

[37] R. O'Donnell, D. Breen, S. Wilson, and R. Djukanovic, "Inflammatory cells in the airways in COPD," Thorax, vol. 61, no. 5, pp. 448-454, 2006.

[38] K. Górska, M. Maskey-Warzechowska, and R. Krenke, "Airway inflammation in chronic obstructive pulmonary disease," Current Opinion in Pulmonary Medicine, vol. 16, no. 2, pp. 89-96, 2010.

[39] J. A. Falk, O. A. Minai, and Z. Mosenifar, "Inhaled and systemic corticosteroids in chronic obstructive pulmonary disease," Proceedings of the American Thoracic Society, vol. 5, no. 4, pp. 506512,2008 .

[40] B. Gigante, A. M. Bennet, K. Leander, M. Vikström, and U. de Faire, "The interaction between coagulation factor 2 receptor and interleukin 6 haplotypes increases the risk of myocardial infarction in men," PLoS ONE, vol. 5, no. 6, Article ID el1300, 2010 .

[41] B. Gigante, M. Vikström, L. S. Meuzelaar et al., "Variants in the coagulation factor 2 receptor $(\mathrm{F} 2 \mathrm{R})$ gene influence the risk of myocardial infarction in men through an interaction with interleukin 6 serum levels," Thrombosis and Haemostasis, vol. 101, no. 5, pp. 943-953, 2009.

[42] M. D. McDowall, M. S. Scott, and G. J. Barton, "PIPs: human protein-protein interaction prediction database," Nucleic Acids Research, vol. 37, no. 1, pp. D651-D656, 2009.

[43] Q. Li, C. Wang, Q. Zhang, C. Tang, N. Li, and J. Li, “The role of sphingosine kinase 1 in patients with severe acute pancreatitis," Annals of Surgery, vol. 255, no. 5, pp. 954-962, 2012. 


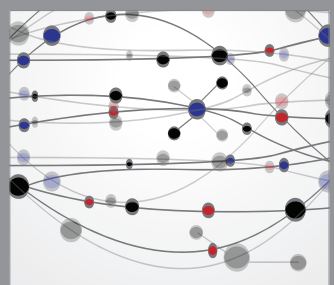

The Scientific World Journal
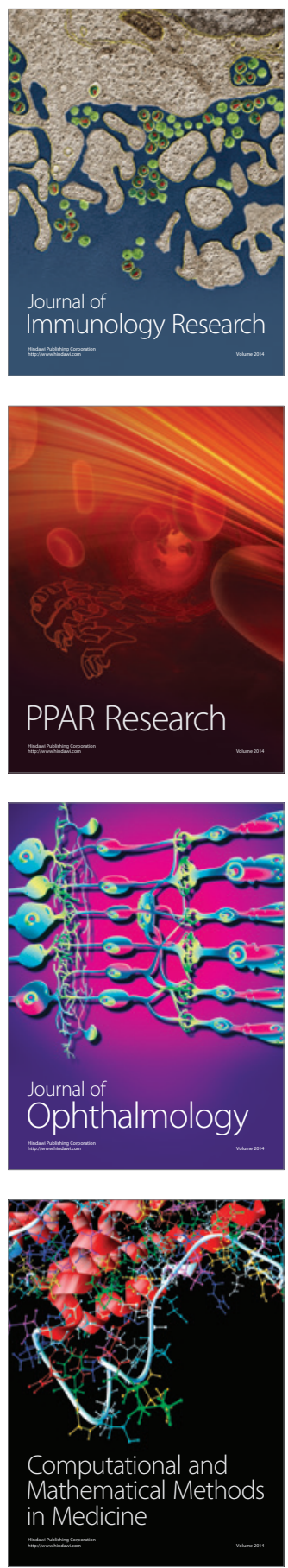

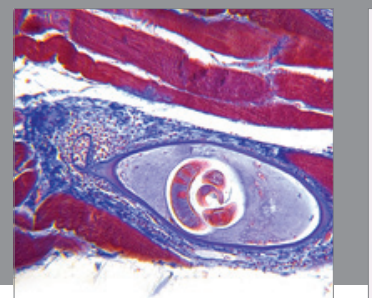

Gastroenterology

Research and Practice
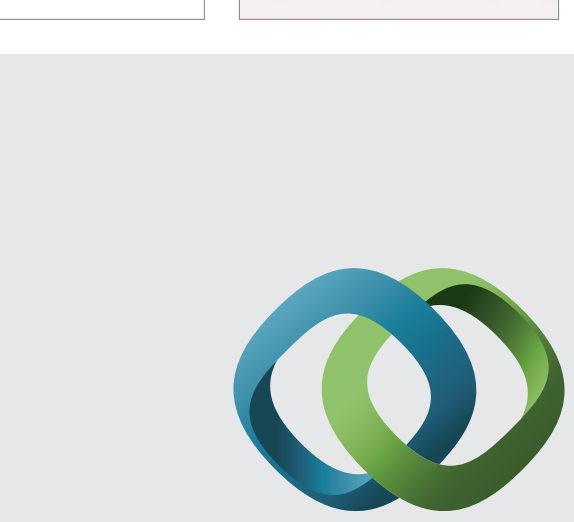

\section{Hindawi}

Submit your manuscripts at

http://www.hindawi.com
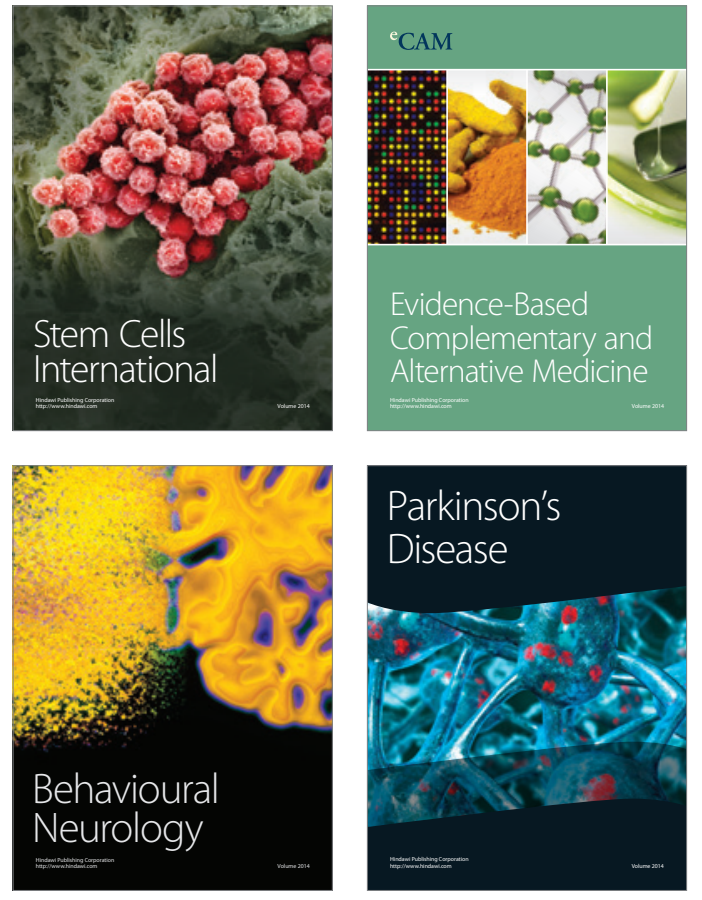
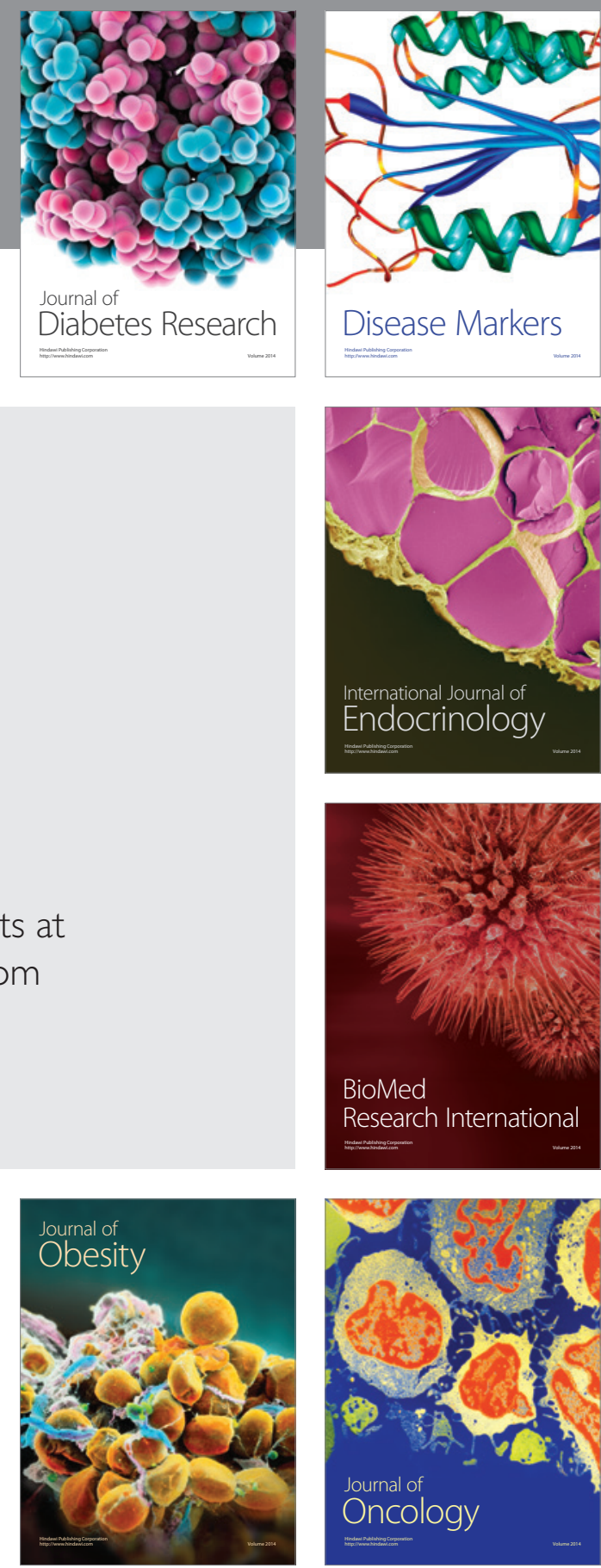

Disease Markers
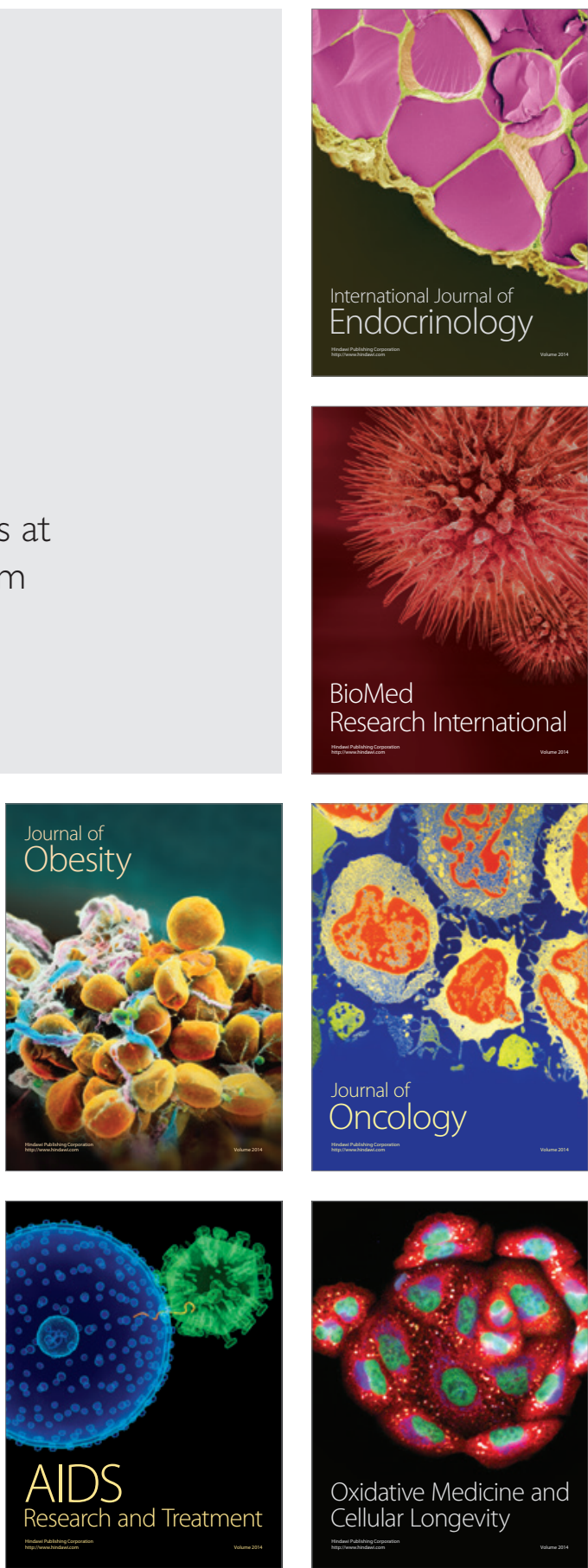